\title{
Mesh distortion immunity of finite elements and the best-fit paradigm
}

\author{
G PRATHAP $^{1}$, V SENTHILKUMAR $^{1}$ and S MANJU ${ }^{2}$ \\ ${ }^{1}$ CSIR Centre for Mathematical Modelling and Computer Simulation \\ (C-MMACS), Bangalore 560 037, India \\ ${ }^{2}$ National Aerospace Laboratories, Bangalore 560 017, India \\ e-mail: gp@cmmacs.ernet.in
}

MS received 19 July 2006; revised 18 September 2006

\begin{abstract}
It has been known for some time that distorted finite elements produce relatively (and, sometimes, dramatically) poor results. This has been related to the completeness condition. In this paper, we investigate this issue and propose that the abstract mathematical viewpoint represented by the completeness condition is actually a statement of the physical need for a finite element computation to recover accurate stresses in the metric space. This follows from the projection theorem describing finite element analysis which shows that the stresses computed by the displacement finite element procedure are a best approximation of the true stresses at an element as well as global level. The simplest possible element is used to elucidate the principles.
\end{abstract}

Keywords. Mesh distortion; best-fit paradigm; parametric-metric element; projection theorem; three-node bar element.

\section{Introduction}

It has been known from the very early days of finite element research that a distorted finite element is much less accurate than a finite element of regular shape (e.g. a quadrilateral element as compared to a rectangular element). Serious studies of mesh distortion sensitivity date from early investigations of the isoparametric formulation (Stricklin et al 1977; Backlund 1978; Gifford 1979; Arnold et al 2002). It was clearly observed that the early isoparametric plane stress elements (8-node and 9-node based on serendipity and Lagrangian shape functions) performed extremely well for regular meshes based on elements of square or rectangular geometry, but that this degraded rapidly when the elements were distorted.

Studies on mesh distortion sensitivity and immunity were recently renewed by Rajendran and co-workers (Rajendran \& Liew 2003; Ooi et al 2004; Rajendran \& Subramanian 2004). These studies explored the counter-intuitive use of unsymmetric formulations characterized by the use of two separate sets of shape functions, viz., the so-called compatibility (or continuity) enforcing shape functions and the so-called completeness enforcing shape functions. The former are chosen to satisfy exactly the minimum inter- as well as intra-element displacement continuity requirements, while the latter are chosen to satisfy all the (linear and higher 


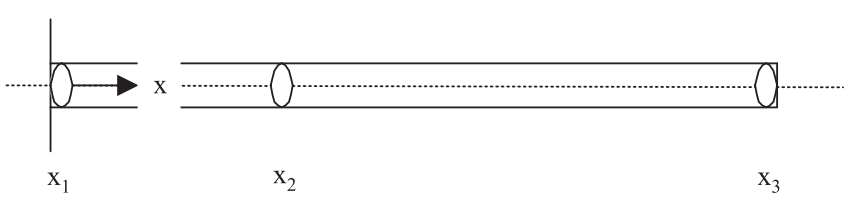

Figure 1. The 3-noded bar element with nodes at $x_{1}, x_{2}$ and $x_{3}$.

order) completeness requirements so as to reproduce exactly a quadratic displacement field. Numerical results from test problems reveal that the new plane stress element (Rajendran \& Liew 2003) is capable of reproducing exactly a complete quadratic displacement field under all types of admissible mesh distortions, only if the continuity enforcing shape functions are based on isoparametric functions and are actually the test functions while the completeness enforcing shape functions are based on metric forms and are now the trial functions. The physical insight into why the test functions should be continuity enforcing and why this is ensured if isoparametric functions are adopted is easy to understand (Rajendran \& Subramanian 2004). Similarly, it is easy to appreciate that if the trial functions are set up using metric functions, the completeness conditions are met (Rajendran \& Subramanian 2004). However, this completeness-fulfilling argument remains at an abstract mathematical level and it is not clear what physically intuitive condition is being satisfied.

Prathap and co-workers (Prathap 1993; Prathap \& Mukherjee) have proposed that variationally correct (i.e no variational crimes are committed) finite element computation automatically seeks out stresses in a best-fit manner (the computed stresses are a best-approximation of the actual stresses) at the element level. This is stated mathematically as a projection theorem (Strang \& Fix 1973) or as an orthogonality condition (Babuska \& Stroubolis 2001) at the global level. Recently Prathap \& Mukherjee $(2003,2004)$ have shown that the best-fit nature is valid at the global level because it is indeed valid at the element level, the global best-fit emerges holistically out of the local best-fits. It is tempting now to propose that the reason why the unsymmetric parametric-metric formulation is effective is because the stress representation is managed in the metric space and this is what is expected of the best-fit paradigm of finite element computation.

We propose to use a very simple 3-noded bar element to seek out the physical insight to explain why the parametric-metric (PM) version has excellent mesh distortion immunity.

\section{The 3-noded bar element using parametric and metric functions}

Figure 1 shows the 3-noded bar element of length $L$ with nodes at $x_{1}, x_{2}$ and $x_{3}$. We assume that the node $x_{2}$ is not at the centre of the bar so that the distortion parameter $\cap=x_{2}-L / 2$. The parametric (better known as isoparametric) formulation requires the interpolation of the $x$ coordinate using $x=N_{i} x_{i}$ and the displacement field $u=N_{i} u_{i}$, where $N_{i}$ are the quadratic interpolation parametric functions, $x_{i}$ are the nodal coordinates and $u_{i}$ is the nodal displacement vector.

If $g$ is the natural coordinate so that $x_{1}$ is at $g=-1, x_{2}$ is at $g=0$ and $x_{3}$ is at $g=1$, then $x$ and $u$ are interpolated by

$$
\begin{aligned}
& x=g(g-1) / 2 x_{1}+\left(1-g^{2}\right) x_{2}+g(g+1) / 2 x_{3}, \\
& u=g(g-1) / 2 u_{1}+\left(1-g^{2}\right) u_{2}+g(g+1) / 2 u_{3} .
\end{aligned}
$$


Although a quadratic interpolation is assumed for the displacement field, we note that when node $x_{2}$ is not at the centre of the bar so that the distortion parameter $\cap$ is non-zero, the strain $\varepsilon=\mathrm{d} u / \mathrm{d} x$ is no longer a linear function of $x$.

Indeed we have,

$\varepsilon=\mathrm{d} u / \mathrm{d} x=\left[\left(u_{3}-u_{1}\right) / 2+g\left(u_{1}+u_{3}-2 u_{2}\right)\right] /\left[\left(x_{3}-x_{1}\right) / 2+g\left(x_{1}+x_{3}-2 x_{2}\right)\right]$

where the denominator represents the Jacobian governing the transformation from $x$ to $g$ spaces. If $x_{2}$ is not exactly mid-way between $x_{1}$ and $x_{3}, J$ is no longer a constant and this has curious implications for the accuracy of the element and this is what we shall investigate next.

Rajendran and co-workers (Rajendran \& Liew 2003; Ooi et al 2004; Rajendran \& Subramanian 2004) proposed that distortion immunity is obtained if the interpolation for the real strain/stress is derived from trial functions in the metric, i.e. $x$ space. The metric part of the formulation now uses $u=M_{i} u_{i}$, where $M_{i}$ are the metric functions. It is a simple exercise to derive these functions (Rajendran \& Subramanian 2004).

\subsection{Virtual strains vs. real stresses}

We simplify the weak form of the statement of the boundary value problem of the onedimensional bar under the action of a body force $f$ as follows:

$$
\int\left(\delta \varepsilon_{v} \sigma-\delta u_{v} f\right) \mathrm{d} x=0 .
$$

The real stress tensor is $\sigma$ and $\delta u_{v}$ and $\delta \varepsilon_{v}$ are the variations of the virtual displacement and strain. We do not now postulate that $\sigma=\mathbf{D} \varepsilon_{v}$ where $\mathbf{D}$ is the constitutive matrix, to emphasise that in the virtual work form in which Eq. (3) is expressed, the real stresses $\sigma$ and the virtual strains $\varepsilon_{v}$ can be introduced independently without violating the variational correctness norms. In other words, the Petrov-Galerkin formulation where the virtual strains are derived from one set of test functions and the real stresses are derived from an independent set of trial functions along with the constitutive law is variationally correct even though a finite element implementation will lead to unsymmetric matrices. Following Ooi et al (2004), for the virtual strain to be admissible, the virtual displacement must be at least $C^{0}$ continuous along the element boundary (inter-element continuity). This is satisfied by the parametric choice of functions for the virtual displacements for any admissible shape of the element (this distinction is not important for the one-dimensional element used here but would become significant when two- and three-dimensional problems are taken up). However, metric shape functions satisfy the inter-element continuity requirement only when the element conforms to certain regular shapes. Let us now examine the suitability of the parametric or metric functions for the displacement fields $u$ from which the real stresses $\sigma=\boldsymbol{D}(\nabla u)$ is computed, where $\nabla$ is the strain-displacement operator. It becomes immediately clear, from reference to (1), that the real stresses are those stresses which manage to represent the equilibrium of forces. It is obvious that in the real world (which is metric), this is best represented in metric space. Thus a finite element formulation will seek out stresses $\sigma^{h}$ which are a best-fit in the metric space of $\sigma$.

Note that if we do not make this distinction between real and virtual, i.e. if $\varepsilon=\varepsilon_{v}$ because identical functions are used for the trial and test functions in the finite element formulation of the problem, we get the classical Galerkin formulation and consequently symmetric matrices. Since it is paramount that the virtual displacements must be in parametric form to ensure inter-element continuity, a Galerkin formulation will find it difficult to achieve the best-fit 
stress paradigm as then the real stresses are also represented in the parametric space and this may not transform in a simple affine manner to the metric space. Another physically intuitive way of looking at this situation is that the trial functions define the real stresses satisfying the kinetic (equilibrium) condition in the virtual work equation and the test functions enforce the kinematic conditions of continuity. The Petrov-Galerkin approach allows the competing requirements of managing continuity and equilibrium under mesh distortion to be negotiated. This explains why the unsymmetric PM formulation gives the best distortion tolerance (Rajendran \& Liew 2003; Ooi et al 2004; Rajendran \& Subramanian 2004). We choose the numerical experiments carefully to highlight this aspect clearly.

\section{Formulation of PP and PM versions of the 3-noded bar element}

Rajendran and co-workers have given a very elaborate account of the formulation of the unsymmetric problem (Rajendran \& Liew 2003; Ooi et al 2004; Rajendran \& Subramanian 2004). For the sake of completeness, this is summarized as shown below. The parametric shape functions are defined as

$$
N_{1}=g(g-1) / 2, \quad N_{2}=\left(1-g^{2}\right) \text { and } N_{3}=g(g+1) / 2 .
$$

From this, the strain displacement matrices can be written as

$$
\boldsymbol{B}_{\boldsymbol{p}}=\left[N_{1, g}, N_{2, g}, N_{3, g}\right] / x_{, g} .
$$

The metric functions $M_{i}$ can be easily derived (Rajendran \& Subramanian 2004) and the corresponding strain displacement matrices are

$$
\boldsymbol{B}_{\boldsymbol{m}}=\left[M_{1, x}, M_{2, x}, M_{3, x}\right] .
$$

The stiffness matrices for the PP and PM elements are then

$$
\boldsymbol{K}_{p p}=\int\left(\boldsymbol{B}_{p}\right)^{\boldsymbol{T}} \boldsymbol{D} \boldsymbol{B}_{p} \mathrm{~d} x
$$

and

$$
\boldsymbol{K}_{p m}=\int\left(\boldsymbol{B}_{p}\right)^{\boldsymbol{T}} \boldsymbol{D} \boldsymbol{B}_{m} \mathrm{~d} x .
$$

The consistent load vector for both cases is identical and is given by

$$
\boldsymbol{f}_{p p}=\boldsymbol{f}_{p m}=\int(\boldsymbol{N})^{\boldsymbol{T}} \boldsymbol{b} \mathrm{d} x,
$$

where $\boldsymbol{b}$ is the body force. In (7) to (9), the cross-sectional area has been taken as unity.

\section{A numerical experiment with the 3-noded bar element}

\subsection{Case of uniformly distributed load $q=1$}

We take up a single element test where the bar is fixed at $x_{1}=0$ and is free at $x_{3}=1$. The length of the bar is therefore $L=1$. Young's modulus is taken as $E=1$, area of crosssection $A=1$, and a uniformly distributed axial load of intensity $q=1$ is assumed to be 
Table 1. Deflections $u_{2}$ and $u_{3}$ for a single-element test of fixed-free bar with uniform traction load of $q=1$.

\begin{tabular}{|c|c|c|c|c|c|c|}
\hline \multirow[b]{2}{*}{ Displacements } & \multicolumn{3}{|c|}{$\cap=0$} & \multicolumn{3}{|c|}{$\cap=-0.05$} \\
\hline & PP & PM & Exact & PP & PM & Exact \\
\hline$u_{2}$ & $0 \cdot 37500$ & $0 \cdot 37500$ & 0.37500 & $0 \cdot 34833$ & $0 \cdot 34875$ & $0 \cdot 34875$ \\
\hline$u_{3}$ & $0 \cdot 50000$ & 0.50000 & 0.50000 & $0 \cdot 50000$ & 0.50000 & $0 \cdot 50000$ \\
\hline
\end{tabular}

applied along the length of the bar. The units are assumed to be consistent so that we have $\sigma=\varepsilon=1.0-x$. Note that the stress and strain are defined in a very simple linear fashion in the metric space, and if the element is distorted, this would be captured only in a nonlinear fashion by the parametric coordinate system. Indeed, we then have

$$
\sigma=\varepsilon=1 \cdot 0-x=1.0-x_{2}-0.5 g+\cap g^{2} .
$$

Two elements are developed: the PP element uses parametric interpolations for both test and trial functions, while the PM element uses parametric interpolations for the test functions and metric interpolations for the trial functions.

Table 1 displays the results from a single-element model of the example. We find that the PP and PM elements give the exact displacements when there is no distortion $(\cap=0)$. When there is distortion $(\cap=-0.05)$, only the PM formulation is insensitive to distortion. To understand why this is so, we must study the stress/strain recovery more carefully. Figure 2 shows the stress/strain recovered from the displacements computed using the PP element for the case with distortion, where a 2-pt. integration is used to evaluate the stiffness matrix.

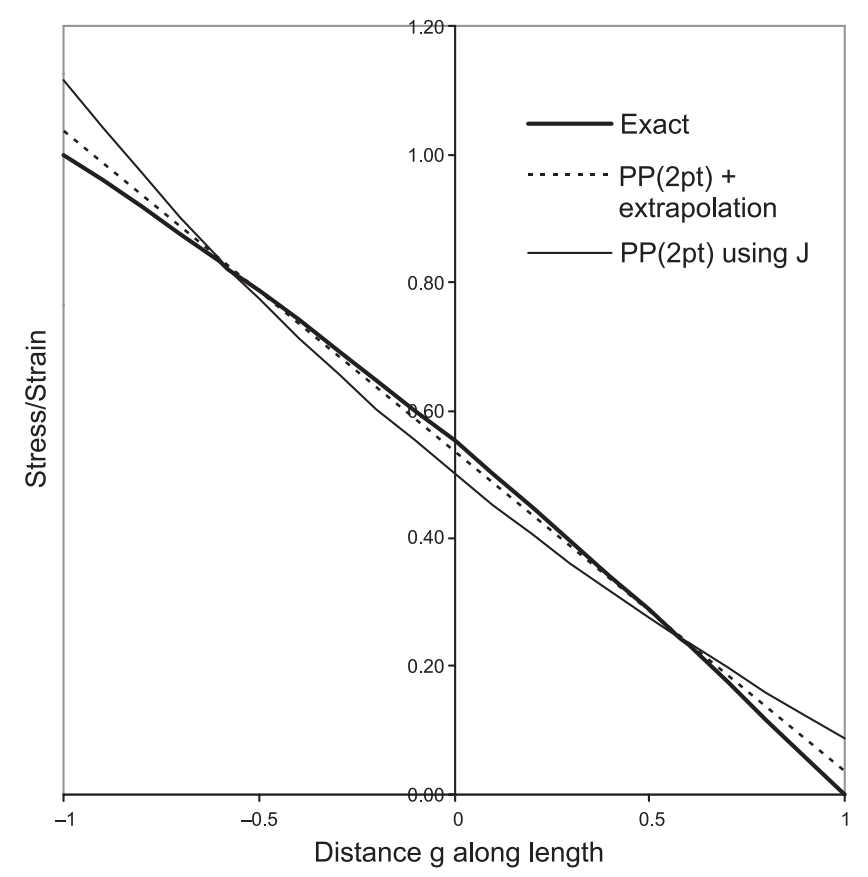

Figure 2. The variation of stress in a fixed-free bar for uniformly distributed load when $x_{2}=0.45$ for PP model. 


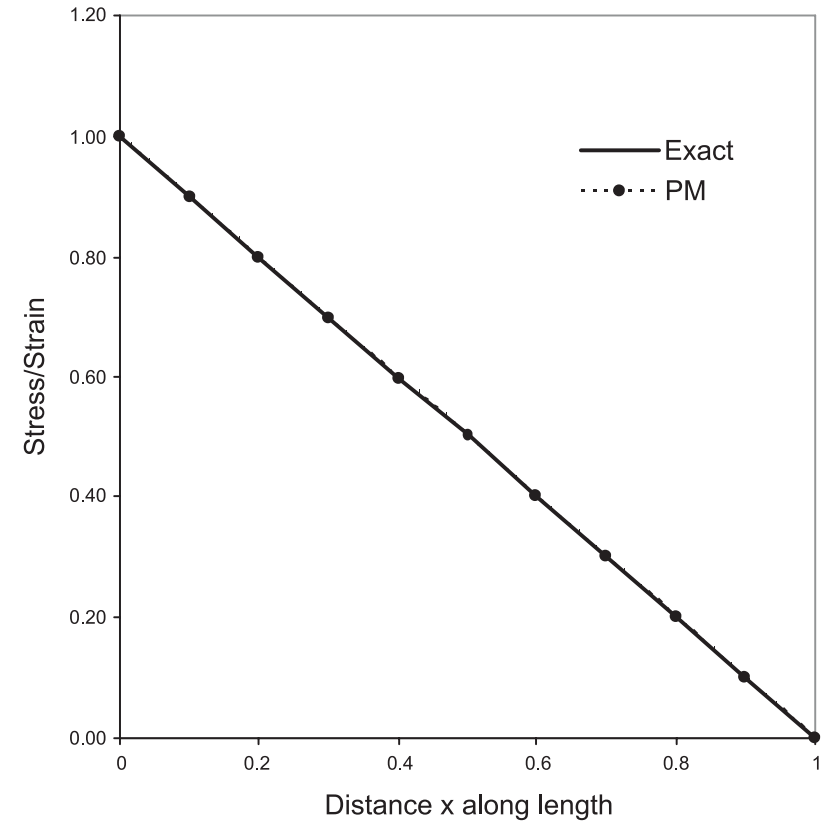

Figure 3. The variation of stress in a fixed-free bar for uniformly distributed load when $x_{2}=0.45$ for PM model.

From figure 2, we see that if the strain/stress is computed using the parametric transformation involving the Jacobian $J$ in the denominator, the variation of strain thus computed is as shown by the thin solid line. Due to the rapid variation of $J$ over the element length, it is not possible for it to sensibly capture the linear variation in the metric space (shown by the thick solid line in the $g$ space). The dashed line indicates the extrapolation of stress from the Gauss point values used for the 2-pt. Gaussian numerical integration of the PP stiffness matrix. This is a closer approximation of the actual variation of strain and explains why the 2-pt rule is favoured in most industry standard packages. For a more definitive closure between predictions and computations, we rigorously derive the various relationships in the next section.

Figure 3 now shows the stress/strain recovered from the displacements computed using the PM element for the case with distortion. Again, a 2-pt. integration is used to evaluate the stiffness matrix. From figure 3, we see that the strain/stress computed using the metric functions is exactly the same as the exact solution. The Jacobian $J$ is no longer in the picture. With the PM formulation, it is now possible to sensibly capture the linear variation in the metric space (shown by the thick solid line in the $x$ space).

\subsection{Case of quarter point singularity when $g=-0 \cdot 5$}

Table 1 and figures 1 and 2 show the result when the distortion is $\cap=-0.05$. In fact, for whatever distortion $(\cap=-0.5$ to $\cap=-0.5)$ the PM formulation is able to recover the linear variation of stresses exactly. This includes the curious case of the quarter point singularity, where when $g=-0.5$ the PP formulation produces singular stresses. However, the PM formulation has no difficulty here and recovers the correct stress pattern. Indeed, for this case, $u_{2}=0.2187464$ and $u_{3}=0.5$ give the computed stress as $(1-x)$ ! 
Stresses from PM model and Best-Fit rule

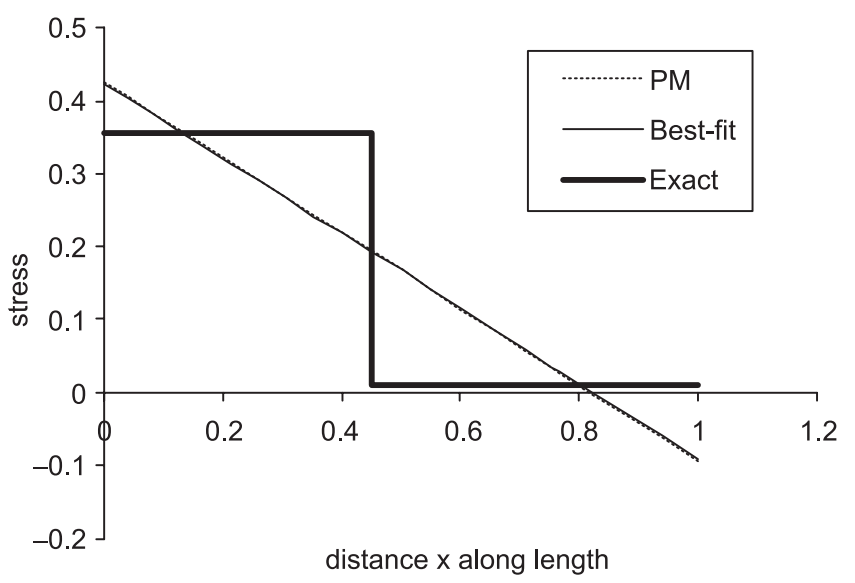

Figure 4. The variation of stress in a fixed-free bar for varying distributed load when $x_{2}=0.45$ for PM model.

\subsection{Case of varying distributed load $q=1-x$}

We now take up the same single element test as before but impose a more complicated loading case, that of a varying distributed load of intensity $q=1-x$. The exact solution for this leads to a cubic polynomial for the displacement field and a quadratic variation for the stress field. However, this can never be realized in a finite element computation because of the need to replace the loading by a consistent load vector. The use of a consistent load vector to replace this system is equivalent to the imposition of concentrated loads $F_{i}$ at the three nodes, and the stress variation corresponding to this is of a stepped nature as seen by the solid line in figure 4 . The loads can be quite easily computed by the standard procedure shown in (9). The units are assumed to be consistent so that we have $\sigma=\varepsilon=F_{2}+F_{3}$, for $x<x_{2}$ and $\sigma=\varepsilon=F_{3}$ for $x>x_{2}$.

We investigate the computed results obtained using the PM element model to see the extent to which the best-fit property is preserved for this case. Figure 4 shows the variation of stresses computed from the displacements from the PM model as compared to the exact stress (solid line $)$ in the bar when the concentrated loads are placed at $x_{2}=0.45(\cap=-0.05)$ and $x_{3}=1.0$ corresponding to the varying distributed load of intensity $q=1-x$. The load at $x_{1}=0$ is obviously ineffective due to the Dirichlet condition imposed there. The PM model, due to the use of the $M$-trial functions, gives a linear variation of stress. The solution is seen to be stable, implying that the stability constant (inf-sup constant) ensures the best approximation and that this is stable. Also shown for comparison is the stress from best-fit approximation (shown by the thin solid line) obtained from the actual stepped stress variation (thick solid line) the finite element model is trying to represent. The stress from PM (thin broken line) is very slightly in error. Indeed, as the distortion increases, this discrepancy increases. This is actually due to the fact that the PM formulation is a best-fit of the PP formulation of the problem and not the best-fit of the exact stress that is being modelled. The analysis relevant to this is too detailed to be given here and is presented elsewhere (Prathap et al 2005). Figure 5 shows the variation of stresses computed from the displacements from the PM model as compared to the "exact" stress (solid line) in the bar when the node $N_{2}$ is placed at $x_{2}=0 \cdot 35(\cap=-0 \cdot 15)$ for the same problem with the system of concentrated loads equivalent to the linearly varying distributed load. Now there is a noticeable difference between the stress computed by the PM 
Stresses from PM model and Best-Fit rule

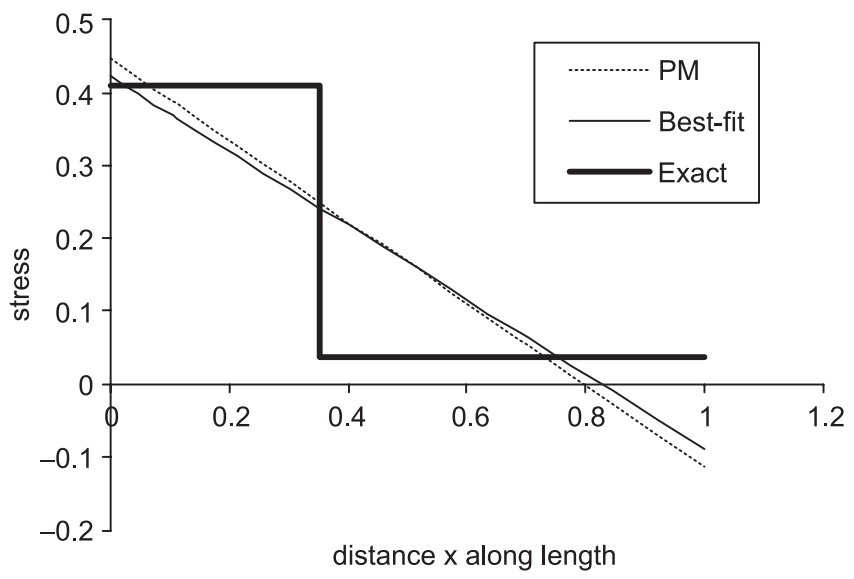

Figure 5. The variation of stress in a fixed-free bar for varying distributed load when $x_{2}=0.35$ for PM model.

model and the best-fit. Indeed, it can be shown (Prathap et al 2005) that the PM model is a best-fit to the PP model.

\section{Closure between predictions and computations}

For scientific closure, what is observed phenomenologically (in this case, computed by the finite element model) must be independently arrived at by prediction from first principles. With particular reference to the results from the PP model (table 1 and figure 2), we must be able to demonstrate that the stress computed using the strain-displacement relations and the computed nodal displacements is the same as predicted by the application of the best-fit rule to the anticipated exact stress.

From table $1, u_{2}=0.34833$ and $u_{3}=0.5$ gives the computed strain as,

$$
\varepsilon_{c}^{h}=(0.5-0.3932) /(1+0 \cdot 2 g),
$$

where the denominator represents the Jacobian defining the transformation from the parametic $g$ to the metric $x$ space. It is this function that is drawn as the thin solid line in figure 2 . As a 2-pt. Gaussian integration rule has been employed, the strain sensed by the finite element computation is linear in $g$ and can easily be extracted from (11) by extrapolation from the two points corresponding to the 2-point Gauss-Legendre integration rule, $g_{i}= \pm 1 \sqrt{3}$ as

$$
\varepsilon_{c}^{h}(2-p t)=0.5333-0.5 g .
$$

This is indicated by the dashed line in figure 2 .

Let us now predict this from an application of the best-fit rule to the exact strain which is defined in metric space as

$$
\begin{aligned}
\varepsilon & =1.0-x \\
& =1.0-x_{2}-0.5 g+\cap g^{2} \\
& =0.55-0.5 g-0.05 g^{2} \\
& =0.5333-0.5 g+0.0166\left(1-3 g^{2}\right) .
\end{aligned}
$$


We have deliberately expanded it in terms of Legendre polynomials so that we can sense directly that the predicted best-fit in the $g$ space to this is obtained as the straight line passing through the points $g_{i}= \pm 1 \sqrt{3}$ (a property emerging from the orthogonal nature of the Legendre polynomials) as,

$$
\varepsilon_{p}=0.5333-0.5 \mathrm{~g}
$$

The exact agreement between the computed $\varepsilon_{c}^{h}(2-p t)$ and the predicted $\varepsilon_{p}$ now provides us the scientific closure.

The reason for the small discrepancy between the exact displacement $u_{2}$ and that computed by the PP model is due to the fact that the exact strain in the metric $x$ space has been replaced by a linear strain in the parametric $g$ space. The PM model does not suffer from this deficiency because the exact strain is now a linear function in the metric space and this is exactly captured by the finite element strain which is also a linear function in $x$.

\section{Unsymmetric formulation and the inf-sup condition}

The inf-sup condition is a fundamental requirement for stability and optimality of conventional finite element schemes where there is locking, or unconventional schemes (where there is varaiational incorrectness, e.g. due to reduced integration and consequently zeroenergy modes, or for mixed or hybrid finite element schemes, Iosilevich et al 1997). In the present case, the abstract formulation has to be examined on this Babuska-Brezzi condition. Where only trial functions are used, the error is (up to the multiplicative constant $C$ ) the error of the best approximation of the exact solution by the trial functions. Where both trial and test functions are used, it is speculated that the trial functions are related to the approximation (approximability) and the test function to the stability (Arnold \& Babuska 1984).

From the foregoing, it is accepted that when the inf-sup condition is satisfied, the element has an optimal (maximum) convergence rate. Following Bathe et al (2000), the smallest non-zero eigenvalue of the problem can serve as a surrogate for the inf-sup condition of the problem. Dynamic studies using the PM formulation (to be communicated separately) show that the log of the smallest non-zero eigenvalue is a constant when plotted against the log of the characteristic element length, thereby confirming that the PM formulation has an optimal convergence rate.

\section{Conclusions}

This paper confirms that in finite element modelling, the best-fit paradigm gives an accurate picture of how strains and stresses are computed, even when there is distortion in the element leading to a non-uniform mapping from the parametric to metric space. This paradigm also elegantly explains why the PM formulation, as observed more generally even for $2 \mathrm{D}$ and $3 \mathrm{D}$ applications, is immune to mesh distortion (Rajendran \& Liew 2003; Ooi et al 2004; Rajendran \& Subramanian 2004). It is reasonable to conclude that the unsymmetric parametricmetric formulation is effective because the stress representation is managed in the metric space and this is what is expected from the best-fit paradigm of finite element computation. 
The authors benefited greatly from intensive discussions with Dr S Rajendran of the Nanyang Technological University, Singapore. The authors also thank the anonymous referees for many helpful suggestions to improve the paper.

\section{References}

Arnold D N, Babuška I, Osborn J 1984 Finite element methods: Principles for their selection. Comput. Meth. Appl. Mech. Eng. 45: 57-96

Arnold D N, Boffi D, Falk R S 2002 Approximation by quadrilateral finite elements. Math. Comput. 71: 909-922

Babuška I, Stroubolis T 2001 The finite element method and its reliability (Oxford: Clarendon)

Backlund J 1978 On isoparametric elements. Int. J. Numer. Meth. Eng. 12: 731-732

Bathe K J, Iosilevich A, Chapelle D 2000 An inf-sup condition for shell finite elements. Comput. Struct. 75: 439-456

Gifford L N 1979 More on distorted isoparametric elements. Int. J. Numer. Meth. Eng. 14: 290-291

Iosilevich A, Bathe K J, Brezzi F 1997 On evaluating the inf-sup condition for plate bending elements. Int. J. Numer. Meth. Eng. 40: 3639-3663

Ooi E T, Rajendran S, Yeo J H 2004 A 20-node hexahedron element with enhanced distortion tolerance. Int. J. Numer. Meth. Eng. 60: 2501-2530

Prathap G 1993 The finite element method in structural mechanics (Dordrecht: Kluwer Academic)

Prathap G, Mukherjee S 2003 The engineer grapples with theorem 1.1 and lemma 6.3 of Strang and Fix. Curr. Sci. 85: 989-994

Prathap G, Mukherjee S 2004 Management-by-stress model of finite element computation. Research Report CM 0405, CSIR Centre for Mathematical Modelling and Computer Simulation, Bangalore, November

Prathap G, Manju S, Senthilkumar V 2005 The unsymmetric formulation and variational correctness. Research Report CM 0506, CSIR Centre for Mathematical Modelling and Computer Simulation, Bangalore, June

Rajendran S, Liew K M 2003 A novel unsymmetric 8-node plane element immune to mesh distortion under a quadratic field. Int. J. Numer. Meth. Eng. 58: 1718-1748

Rajendran S, Subramanian S 2004 Mesh distortion sensitivity of 8-node plane elasticity elements based on parametric, metric, parametric-metric, and metric-parametric formulations. Struct. Eng. Mech. 17: 767-788

Strang G, Fix G J 1973 An analysis of the finite element method (Englewood Cliffs, NJ: Prentice-Hall)

Stricklin J A, Ho W S, Richardson E Q, Haisler W E 1977 On isoparametric vs. linear strain triangular elements. Int. J. Numer. Methods Eng. 11: 1041-1043 